\title{
The Challenge of Electromagnetic Field Measurements for Modern 5G Devices
}

\author{
Manuel Sierra Castañer ${ }^{1}$ \\ (1) Centro de Investigación en Procesado de la Información y Telecomunicaciones, ETSI Telecomunicación. Universidad \\ Politécnica de Madrid, 28040, Madrid, España, manuel.sierra@upm.es.
}

\begin{abstract}
This overview paper shows the challenges for antenna measurements facing with $5 G$ devices. The new antenna related technologies: higher frequency bands to millimeter bands, broadband, new technologies as distributed MIMO, massive MIMO, phased arrays, adaptive beamforming or the fact that the sensors are fully integrated to the telecommunication system makes a real challenge the characterization of the antennas themselves. The figure of merit of interest for the users will be much more complicated than the far field performance. In fact, much of these devices will be interconnected in near field, not only in far field, and basically the interest will come in the reduction of the bit error rate and the increase of the capacity. All these facts make necessary to use Over the Air measurement systems, increasing the measurement speed (real time) and increasing the accuracy for measuring the millimeter frequencies.
\end{abstract}

Index Terms-5G, measurement, near field, sensors.

\section{INTRODUCTION}

This paper presents a revision of the required changes in antenna measurement systems and techniques to face the emerging antenna technologies related with 5G. 5G means lower latency, lower energy consumption, higher speed connection, higher availability and higher number of users (people and machine). All these facts imply more complicated devices, and particularly, active, multiple and distributed antennas. New and complex antenna technologies as phased arrays, distributed MIMO, massive MIMO, smart antennas require different antenna (or better system) measurement techniques to be able to extract the most important parameters that define the physical layer of our devices. However, this is not new, the continuous and rapid evolution of the personal communications have pushed the rapid evolution of the measurement systems. The most important point now is that the number of devices to be characterized will be huge, and the measurement systems will have to be efficient, very fast, precise and cheap.

This paper is not a research paper, but an overview one to open the convened session about $5 \mathrm{G}$ measurements. In this sense, I have divided the contents in the following sections: section II makes an overview of the most important antenna technologies, understood in a general way. Section III reports on the evolution of measurement systems to face with this challenge. Section IV summarizes the required improvements in the definition of antenna measurement technology (including both systems and parameters) to characterize the physical layer of the communication systems. Finally, some conclusions are extracted in Section V.

\section{NEW ANTENNAS FOR 5G STANDARD}

This section reviews the most important features concerning the antennas and radiofrequency subsystems for the $5 \mathrm{G}$ standards that will affect to the measurement systems:

\section{A. Higher frequency bands:}

The spectrum allocated by ITU-R for $5 \mathrm{G}$ services will go up to $100 \mathrm{GHz}$ (and probably higher in future evolution). This means new devices will be extensively commercialized in a close future in millimetre wave frequency bands.

\section{B. Broadband services:}

Also, the efficient use of the spectrum will be the main challenge for $5 \mathrm{G}$ operators. Very wide bands with an efficient use of the spectrum will be necessary for $5 \mathrm{G}$. The measurement systems will deal with wideband systems instead of narrow band systems as we are used to. Also, antenna performance will be measured including 5G modulated signals.

\section{Compact devices:}

User terminals, either for personal communications or Internet of things (machines), will be compact. It will be impossible to separate antenna from RF system and Over the Air (OTA) measurements are required [1]. The characterization of these terminals has to include both the system parameters and the antenna parameters, but using their specific transmitters and receivers (base station emulators).

\section{Complex Antenna technology for Base Stations}

Antennas won't be more passive elements connected to the rest of the system. Beamforming networks, distributed MIMO, Massive MIMO, adaptive antennas will be common in $5 \mathrm{G}$ base stations (mainly for hot spots). This means that antennas won't have a static radiation pattern. However, they will be able to adjust the radiation characteristics to every user specific situation. The classical far field antenna parameters won't be as useful as they were for passive 
antennas. Also, in most of the cases, there won't be a proper RF connector, making necessary OTA measurements.

\section{E. Near-field applications}

Near field communication systems, as vehicle to infrastructure communication, short range and wideband applications among others, will appear to increase the spectrum efficiency. In these cases, far field parameters will not be useful anymore. Antenna performance will require more information.

\section{REVIEW of Antenna MeAsurement TeChniques FOR 5G}

It is clear that OTA measurements are necessary for $5 \mathrm{G}$ measurements. Traditional far field antenna measurements, or the extraction or far field antenna parameters through near field measurements, don't seem the most appropriate case for characterizing 5G complex systems. Real environment measurements, as drive tests, are of course necessary for testing the performance of the network, but they are costly expensive and not repeatable. Therefore, they cannot substitute laboratory tests for the RF chain (including antennas).

During the last years, different alternatives have been studied for OTA measurements [2]: reverberation chambers, radiated multistage method and pattern measurements, either in far field or near field. In this section, I will review the advances in reverberation chambers, far field, compact antenna ranges and multiprobe antenna measurement systems, and I will also include extreme near field scanners.

\section{A. Reverberation chambers}

Reverberation chambers [3-5] is a metallic cavity that emulates a rich multipath environment, using the movement of mode stirrers during the characterization. The device under test (DUT) is placed inside the cavity and some parameters are evaluated in a non-intrusive way. One of the advantages of this system is that the channel is controlled: a Rayleigh-fading channel with a isotropic scattering, and therefore the measurements are repeatable. In fact, probably this channel is more similar to real life for non line of sight applications. However, it is difficult to extract conclusions valid for all kind of channels.

The parameters characterized using reverberation chambers are radiation efficiency, diversity gain, input impedance of the AUT, MIMO gain and MIMO matrix coefficients, throughput data rate, TIS (sensitivity) and total radiated power.

Also, if the antennas are very large, it becomes difficult to ensure equally distributed field on the antenna area. Also, it is impossible to emulate scenarios for smart antenna characterization.

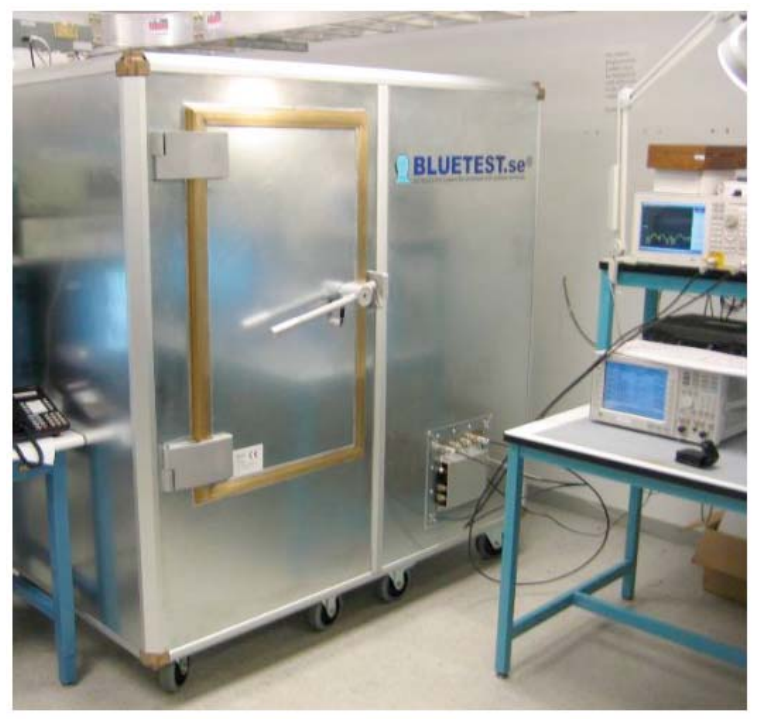

Fig. 1: Bluetest Reverberation Chamber

Finally, it is not possible to characterize the near or the far field using reverberation chambers, while for base station antennas, the channel spatial profile is expected to be closer to line of sight than to isotropic one, making useful the full characterization of the radiation pattern for some applications.

Radiation pattern are measured traditionally in far field systems, compact antenna test ranges and near field systems. If real time measurements are required (for complex antennas as phased arrays this becomes a requirement), classical near field systems are not valid since near to far field transformation requires full sphere, cylinder or plane characterization. For these cases, multiprobe systems are a very good option.

\section{B. Far field systems}

For small size of antennas and specific frequencies, where the measurement distance fulfills $r \geq 2 D^{2} / \lambda$, where D is the maximum dimension of the AUT and $\lambda$ is the wavelength, the antenna pattern can be characterized in reali time using far field systems. Of course, classical far field systems have to be modified to be able to include OTA measurements and other of the aspects mentioned in the previous section: specific $5 \mathrm{G}$ receivers, phase recovery systems (if necessary), wide band probes, millimeter systems...

The main drawback of far field is the limitation of measurement distance, depending of frequency band and antenna size. For large antennas or high frequencies, far field systems become unaffordable. Compact antenna test ranges and near field system are used traditionally to overpass this fact: in the first case real time measurements are done, because of the transformation of the spherical wave in a plane wave using a specific hardware, while in the second case, the near to far field transformation is performed with an algorithm. 


\section{Compact Antenna Test Ranges}

When the required measurement distance becomes very large, a good solution is the use of Compact Antenna Test Ranges (CATR). Usually, CATR are made with a single or dual reflector, based on a parabolic surface for the main reflector. This parabolic surface transforms the impinging spherical wave in a plane wave. The AUT, placed in a specific volume called quiet zone, is excited by this plane wave. Therefore, far field measurements can be done in real time. It is noticeable that for high frequencies and not very large antennas (i.e. 5G devices) CATR can have not very large dimensions as the classical ones (Figure 2).

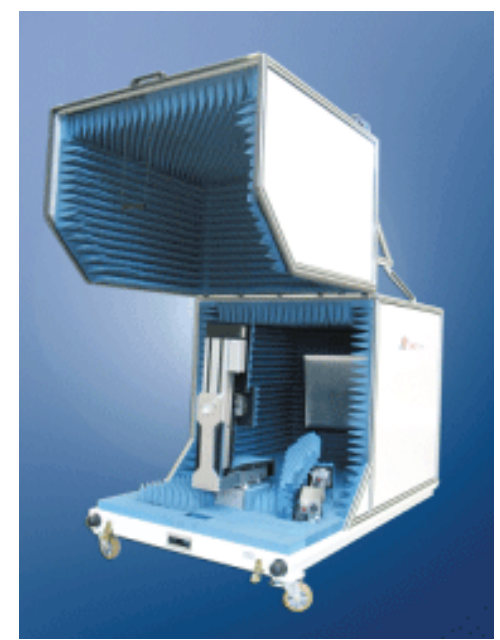

Fig 2: MVG Mini CATR

For 5G measurements, again some changes have to be done with respect the classical measurement set-ups: again OTA measurement set-up and specific $5 \mathrm{G}$ receivers should be used for using CATR with $5 \mathrm{G}$ devices.

\section{Multiprobe antenna measurement systems}

Near field antenna measurement systems [6-7] are a very good option for characterizing passive antennas. When dealing with active antennas, MIMO systems, 5G systems... several drawbacks appear: probably the most important is the measurement time, that makes impossible the real time near field determination for a high number of possible RF states. Near field multiprobe antenna measurement systems (Figure 3) substitute one of the axis by a set of probes, reducing drastically the measurement time, and making possible real time OTA measurements. Different techniques to measure in real time the field in the arch have been studied [8].

During the last year, over the air measurement techniques have been included to this multiprobe system: the measured parameters are not only total radiated power or total isotropic sensitivity but also the equivalent isotropic radiated power and effective isotropic sensitivity for each angular direction. The use of channel emulators allows the characterization of the RF system performance in standard real environments.

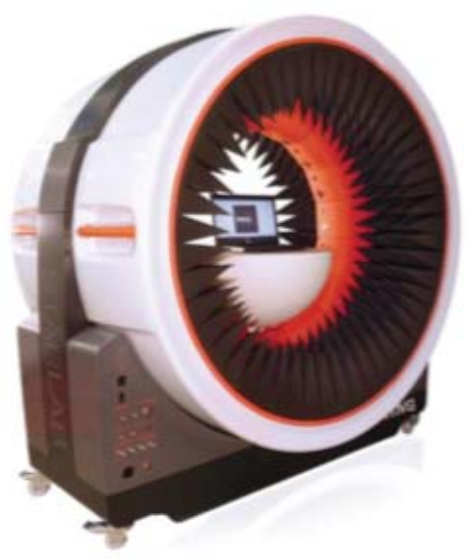

Fig. 3: MVG StarLab multiprobe System

Also, the combination of multiprobe near field techniques with source reconstruction techniques [8] allows the calculation of the sources in the device under test for the different measurement frequencies. Once, these currents are calculated, the radiation properties can be calculated in either near or far field (basically in any point of the space). Also, spurious radiation can be detected. Probably, the main challenge is how to deal with this big amount of information for the device under test.

The main challenges for these systems are the requirement of measuring in near field for large antennas, the cost of the required hardware for the multiprobe systems and the use of the huge information available in these systems.

\section{E. Extreme near field planar scanners.}

Although, it is not considered in the standards wideband extreme near field can be very useful to determine the fields on the apertures of complex devices or base stations. The solution for $5 \mathrm{G}$ measurements proposed by Speag [10] allows the acquisition of amplitude in wideband in two different planes and the extraction of the phase and amplitude of the electric field. Once, this is obtained the near of far field could be determined for any point of the space. The challenges here are how to avoid the interaction between probes and device under test and how to make the acquisition very fast. Probably, the evolution of these systems will have multiprobe planar scanners to combine the velocity of the previous ones with the scan capability of these ones.

In any case, it is clear, that the previous systems have to be completed with $5 \mathrm{G}$ transmitters and receivers, channel emulators, and complex post-processing techniques. The different hardware providers: Keysight [11], R\&S [12], National Instruments [13] among others are already offering equipment to integrate $5 \mathrm{G}$ emulators at mm-wave frequencies, allowing MIMO testing, antenna mutual coupling characterization, active antenna characterization in $\mathrm{mm}$ wave frequencies. These systems have to be combined with the Over the Air measurement systems to be able to 
provide a complete characterization of the new antenna technologies.

However, some issues are still open for the antenna measurement community. Next section presents the most relevant aspects to be considered for facing $5 \mathrm{G}$ antenna measurement challenge.

\section{Challenges for Modern DeVices 5G Field ANTENNA MEASUREMENT SYSTEMS}

5G standard is a real challenge for mobile communications, and this includes antenna measurement manufacturers. The very fast evolution we are facing with $5 \mathrm{G}$ technologies will quickly be integrated also in our measurement systems, changing from the classical test systems to innovative ones. Some of the challenges are mentioned in this section:

- Over the air measurements with $5 \mathrm{G}$ transmitter/receivers and channel emulators. Radio channel of the cellular communication systems are not anymore narrow band and line of sight. The system engineers require optimizing the capacity of the network using all the capabilities of the spectrum. The antennas are not anymore the last or first elements of the transmission or reception chains but another element of the whole system, including the propagation channel.

- How to measure? I mean, under which conditions we have to evaluate our systems, which propagation channels have to be evaluated. Some efforts have been done in this sense, but this has to be clarified. Line of sight will still be a reference, but probably is not the best model for real life.

- Are far field parameters useful enough for 5G testing? A high number of $5 \mathrm{G}$ devices will work in near field, how do we have to represent their performance? Probably, currents information is more appropriate than far field for these applications. In any case, it is necessary to standardize the new parameters to face with new communication systems.

- Low cost and low uncertainty millimetre wave systems. Once new frequency bands were occupied by $5 \mathrm{G}$ systems, there will be a massive production of systems. The measurement systems will be able to use existing technology to drastically reduce the hardware costs and increase the dynamic range.

- Phase recovery systems: the phase of the modulated signals in a very high frequency band will have to be recovered. Algorithms using the information from I/Q demodulators will be used.

- Dealing with huge number of information: new antenna technologies imply infinite possibilities. When dealing with smart antennas, there is not a radiation pattern but infinite sets of antenna configurations. We need to establish some reference situations and give some quality factors. Post processing of all these data will be required, but prior to that, an exact definition of the new quality factors for smart antenna, massive MIMO or the future antenna technologies.

\section{CONCLUSIONS}

This overview paper has examined some of the challenges that antenna measurement engineers and manufacturers will face with $5 \mathrm{G}$. In my opinion, the best approach will be based on over-the-air measurements using near field scanners, with fast and accurate multiprobe scanners, at millimetre wave frequencies, extracting through post-processing as much as possible information of the device under test, and processing it to be able to extract some required quality factors for the new antenna technologies (massive MIMO among others).

And, the technological step that $5 \mathrm{G}$ will provide, will be transferred immediately to other radio systems, meaning a new concept of antenna measurements, much more complex than the one for passive antennas, but also being able to provide much more information of the performance of the system.

\section{ACKNOWLEDGMENT}

Spanish Government supported this work under the project TEC2014-55735-C3-1-R (ENABLING5G, Enabling Innovative Radio Technologies for $5 \mathrm{G}$ networks).

\section{REFERENCES}

[1] "Test plan for wireless device over-the-air perfomance: Method of measurement for radiated RF power and receiver performance". CTIA Certification, Washington, D.C. Tech. Rep. Version 3.5.2. Sept. 2015

[2] W. Fan, I. Carton, P. Kyösti, A. Karstensen, T. Jämsä, M. Gustafsson and G.F. Pedersen "A Step Toward 5G in 2020: Low-cost OTA performance evaluation of massive MIMO base stations" IEEE Antennas\&Propagation Magazine. Vol. 50, No.1, Feb. 2017, pp. 3847

[3] P.-S. Kildal and K. Rosengren, "Correlation and capacity of MIMO systems and mutual coupling, radiation efficiency and diversity gain of their antennas: Simulations and measurements in reverberation chamber", IEEE Communications Magazine, vol. 42, no. 12, pp. $102-$ 112, Dec. 2004.

[4] P. S. Kildal, A. Hussain, X. Chen, C. Orlenius, A. Skårbratt, J. Åsberg, T. Svensson, and T. Eriksson, "Threshold Receiver Model for Throughput of Wireless Devices with MIMO and Frequency Diversity Measured in Reverberation Chamber," IEEE Antennas and Propagation Wireless Letters, vol. 10, pp. 1201-1204, October 2011.

[5] P.-S. Kildal, C. Orlenius, J. Carlsson, "OTA Testing in Multipath of Antennas and Wireless Devices with MIMO and OFDM", invited paper to appear in special issue of Proceedings of the IEEE, July 2012

[6] IEEE Recommended Practice for Near-Field Antenna Measurements. IEEE Std 1720-2012.

[7] A.D. Yaghjian. An Overview of Near-Field Antenna Measurements. IEEE Trans AP. January 1986.

[8] J. C. Bolomey and F.E. Gardiol, "Engineering Applications of the Modulated Scatterer Technique", Artec House, 2001

[9] J. L. A. Quijano, G. Vecchi, "Improved-Accuracy Source Reconstruction on Arbitrary 3-D Surfaces", IEEE Antennas and wireless propagation letters, pp. 4, 2009, Vol. 8, ISSN: 1536-1225.

[10] Speag. "Mastering the Near-Field at 10-110 GHz" www.speag.com.

[11] www.keysight.com

[12] Rhode\&Schwarz. "Test solutions for $5 \mathrm{G}$ to enable your success : Massive MIMO test challenge". www.rohde-schwarz.com/5G

[13] National Insturments. "NI Solutions: Software Defined Radio" www.ni.com $/ 5 \mathrm{~g}$ 